\title{
Osteotomy Model Is Not Suitable To Provide Insight into Normal Bone Healing
}

\author{
${ }^{1}$ Syed Mobashir Yunus, ${ }^{2}$ Farida Ahmad, ${ }^{3}$ Adil Asghar, ${ }^{4}$ Nafis Ahmad Faruqi \\ ${ }_{1,4}^{4}$ Associate Professor and Professor, Dept of Anatomy, JN Medical College AMU Aligarh, UP, India \\ ${ }^{2}$ Associate Professor, Dept of Pharmacology, JN Medical College, AMU Aligarh, UP, India \\ ${ }^{3}$ Assistant Professor, Dept of Anatomy, HIMSR, Jamia Hamdard, New Delhi, India
}

\begin{abstract}
Understanding of biological course of healing is essential for a therapeutic approach and the choice of an adequate animal model can be crucial for the experimental results. Methods: A rabbit tibial osteotomy model with subsequent intramedullary stabilization was performed. The healing progress of the osteotomy model was compared to a closed fracture model. Histological analyses, biomechanical testing and radiological screening were undertaken during the observation period of 84 days to verify the status of the healing process. Results: In contrast to the closed fracture technique osteotomy led to delayed union or nonunion until $84 d$ post intervention. The dimensions of whole reactive callus differed significantly between osteotomized and fractured animals at 28 d post surgery. A lower fraction of newly formed bone and cartilaginous tissue was obvious during this period in osteotomized animals and more inflammatory cells were observed in the callus. Conclusion: The osteotomy technique is associated with cellular and vascular signs of persistent inflammation within the first $28 d$ after bone defect and may be a contributory factor to impaired healing. The model would be helpful to test agents to promote fracture healing but this will not show the normal bone healing pattern.
\end{abstract}

Key words: Callus, Fracture Model, Impaired Bone Healing, Tibial Osteotomy Model,

\section{Introduction}

Coles has reported up to 17 percent of nonunions after treatment of closed tibial shaft fractures. Impairment of fracture healing causes not only individual incapacitation but also produces a negative socioeconomic impact upon society [1]. Hence, by means of in vitro and in vivo experiments, considerable efforts have been made in fracture research to develop therapeutic approaches to accelerate fracture healing. Before promising concepts can be used in humans, it is necessary to verify their efficacy and safety in a variety of animal models. [2] Models simulating impaired fracture healing in animals are difficult to conduct. Animal models of bony nonunion mainly utilize techniques with large segmental defects, unstable fixation or combinations of these procedures [3]. Descriptions or comparative studies of the different methods used in fracture research are often limited in detail. However, understanding of the biological course of healing is essential for a therapeutic approach and the choice of an adequate animal model can be crucial for the experimental results. Furthermore, with respect to laboratory animal stress and avoiding recurrent failure, we believe that critical reports on an in vivo approach are essential.

The regular course of fracture healing can be broken down into different phases: (a) the reactive phase, including fracture, haematoma and inflammation whereby the initial inflammation is regarded as an activator of fracture healing [4]; (b)the reparative phase, characterized by callus formation and lamellar bone deposition, and (c) the remodeling phase, creating the original bone contour.

Impairment of regular osseous healing can result in delayed union or nonunion. Nonunions can be classified as hypertrophic and are termed as vital on the basis of radiological evidence of proliferative external callus formation on the fragment side; if callus formation does not occur, they are termed atrophic [5]. Common reasons for the impaired healing are poor end-to-end contact of the bone fragments, excessive inter-fragmentary movement and poor blood supply due to insufficient vascularization at the site of the defect or the interaction of all factors [6]. Rabbits are the most widely used animal species in bone healing research and osteotomy and closed fracture of long bones are frequently used model techniques [7]. Here we describe the course of delayed healing in an open rabbit tibial osteotomy model in comparison to a closed fracture model with regular healing in order to find a possible explanation for the impairment of healing.

\subsection{Animal model}

II. Material And Methods

Albino rabbits (wt $1.5-2 \mathrm{~kg}$ ) obtained from the central animal house of J.N. medical college were housed and acclimatized at the research animal laboratory and kept on standard pellet diet (Lipton India limited) 
and water ad-libitum. Animal experimentation ethics committee of medical college approved the care and experimental protocol of the study (Registration No. 401/CPCSEA; Dated: 30.03.2010).

\subsection{Surgical protocol and experimental design}

After shaving and disinfection of the right lower leg, the medullary cavity of the tibia was opened at the level of the proximal metaphysis and prepared for stabilization using a $1.5 \mathrm{~mm}$ Kirschner steel wire. Rabbits were anaesthesized by thiopentone sodium (dose $30-50 \mathrm{mg} / \mathrm{kg}$ ) intraperitoneal route then a $1.5 \mathrm{~mm}$ kirschner drilled into tibial shaft percutaneously through tibial tuberosity. The pre-nailed tibial shaft was fractured by an impact device which resulted transverse fracture confirmed by radiograph. Use of $\mathrm{K}$-wire is done to get appropriate alignment and stabilization and prevent comminution at fracture site. The steel wire was removed and osteotomy was performed at the midshaft level of tibia using an impact device by Hiltunen method. [8] The fibula was fractured manually. Osteotomy was stabilized intramedullary with a steel Kirschner wire. After stabilization the wound was closed by a vicryl suture and gentamycin ointment was applied locally. For pain prophylaxis the animals received buprenorhine $(0.05 \mathrm{mg} / \mathrm{kg}$ body weight s.c.) for the first 3 days after the intervention.

The closed fracture of the tibia and fibula was produced in a standardized manner [9] and the stabilization of the fracture was performed in the same manner as described for the osteotomy model with a Kwires. Animals were regularly monitored radiographically. Mediolateral and anterior-posterior radiographs were taken postoperatively and at $28 \mathrm{~d}, 42 \mathrm{~d}$ and $84 \mathrm{~d}$ after surgery.

The animals were sacrificed under anaesthesia by intracardiac injection of calcium chloride.

\subsection{Biomechanics}

For biomechanical testing ipsilateral and contralateral tibiae of osteotomized animals $(\mathrm{n}=18)$ killed at $28 \mathrm{~d}, 42 \mathrm{~d}$ and $84 \mathrm{~d}$ were dissected. The titanium wire was carefully removed under protection of the callus. Proximal and distal to the bone defect the tibia was Technovit-embedded into two moulds (Technovit 9100). Embedding of the contralateral tibia was performed analogously. Each mould was connected to a pivoted axis and the sample was preloaded with an axial force of 5 Newton. A constant linear propulsion $(2 \mathrm{~mm} / \mathrm{min})$, generated by a material testing machine (Zwick 1455), was applied to a lever attached to one of the pivoted axis for transforming the translation of the material-testing machine to a uniform torsional movement. The other side was connected with a strain gauge $(\mathrm{Fmax}=50 \mathrm{~N}, \mathrm{HBM})$ which recorded the torsional moment. Subsequently maximum load, torsional stiffness, and energy absorption were calculated. The data were expressed as percentages of those observed with the contralateral tibia not only to avoid interindividual differences in bone constitution, but also with respect to the curved anatomy and the varying cross section shape of the tibia at different levels. Biomechanical data of $n=18$ animals, which had undergone the fracture technique, were compared to the open osteotomy model.

\subsection{Histology and histomorphometry}

At 28,42 and 84 days after osteotomy ( $\mathrm{n}=6$ each time point) tibiae were carefully removed under protection of the callus bone and fixed for 2 days in $10 \%$ buffered formaldehyde and dehydrated in ascending concentrations of ethanol. At days 5 and 10 bone specimen preparations were fixed in formalin for 2-3 days, decalcified with EDTA, processed in ethanol and xylene and embedded in paraffin. Longitudinal $6 \mu \mathrm{m}$ thick sections were prepared and histological standard staining was performed with Haematoxylin-Eosin (HE).HEstaining was used to quantify the dimensions of reactive callus, to measure the cartilaginous fraction in callus (zones of hypertrophic chondrocytes) and for the purposes of visualizing the fraction of newly formed trabecular bone in callus.

The dimensions of specific regions were measured in micrographs of adjacent slices using the Motic software. With respect to inter-individual varieties, a region of interest (ROI) was established for each preparation i.e. the zone of reactive callus distally and proximally from the centre of bone defect which extended in length 1.5 fold the individual diameter of the cortical bone.

\subsection{Statistics}

Biomechanical data of osteotomized and fractured tibiae (at $42 \mathrm{~d}$ and $84 \mathrm{~d}$ ), dimension of whole callus, zones of reparative trabecular bone and zones of hypertrophic chondrocytes of ROI in both groups (at $7 \mathrm{~d}$ and 28 d) were compared using the Mann-Whitney U-test.

\subsection{Surgical procedure and monitoring}

\section{Results}

The surgical intervention of osteotomy was tolerated by all animals. Up to 3 days after surgery they received pain prophylaxis in one animal there was a slight cranial shift in intramedullary nails. In contrast to the fracture model radiological screening of osteotomized animals revealed neither complete consolidation nor 
original remodelling of cortices until $84 \mathrm{~d}$ after surgery (Fig. 1a,b). The majority of animals showed hypertrophic bone fragments. In a minority of animals osteolytic zones could be observed.

\subsection{Biomechanics}

Biomechanical testing of the tibiae of animals which underwent osteotomy revealed relatively low values for maximal torsional load and stiffness during the observation period (Table 1). In none of the animals did torsional load or stiffness reach comparable values to those recorded for the contralateral tibia; in contrast to the recently collected equivalent data from animals undergoing closed tibial fracture, With respect to the small sample size in the osteotomized group at $28 \mathrm{~d}$, due to unforeseen difficulties and damage when preparing the unstable tibial bone for measurement, the calculation of significances was not applied at this time point. However, the mean values in this group were clearly lower (Table 1).

\subsection{Histological examinations}

Histological screening at 28,42 and $84 \mathrm{~d}$ post surgery revealed microscopically a nonunion of osteotomized tibiae except in 3 out of 6 examined animals at $84 \mathrm{~d}$. These bone samples revealed incomplete union whereby only the periosteal callus was bridged. Histological slices of fractured tibiae revealed bony bridging and status of remodelling until $84 \mathrm{~d}$ (Fig. 2). Early reactive callus, dimensions and cellular compartments were studied. Dimensions of the reactive periostal callus were comparable at $7 \mathrm{~d}$ post osteotomy and fracture, respectively. However, $28 \mathrm{~d}$ after intervention the callus formation of the osteotomized animals was elevated compared to the fractured group (table 2).

The cellular composition of callus consisted mainly of reparative granular cells (fibroblasts), inflammatory cells, chondrocytes and osteocytes or their progenitors and of extracellular matrix in both groups. In osteotomized animals the appearance of inflammatory cells (neutrophils, lymphocytes, multinuclear giant cells) was clearly higher. Early angiogenesis, counting of blood vessels in a defined region of callus revealed similar vessel density at $7 \mathrm{~d}$ post operation in both groups, but significantly higher vessel density in osteotomized animals at $28 \mathrm{~d}$ post bone defect $(\mathrm{p}=0.0034)$. This difference was more pronounced when comparing the anterior tibial sides $(\mathrm{p}=0.001$, Table 3$)$. Almost all of the counted vessels had a luminal diameter of approximately $20 \mu \mathrm{m}$. Early fraction of woven bone and cartilaginous tissue -- Significantly more newly arranged bone was found in fractured animals at $28 \mathrm{~d}$ post operation than in the osteomized group $(\mathrm{p}=0.0091)$. Moreover, in nearly all individuals in both groups, lower amounts of this tissue were observed on the anterior tibial side. This was not only the side with a minor soft tissue envelope but also the side of surgery ( Table 4). More newly formed cartilage was found in the fractured animals 7 and $28 \mathrm{~d}$ post surgery $(\mathrm{p}=0.021)$. Only osteotomized animals accumulated lower amounts of differentiated cartilaginous cells on the anterior tibial side.

\section{Discussion}

In order to evaluate an applicable in vivo model for local therapeutical targeting, we investigated the impaired healing course of a rabbit tibial osteotomy with intramedullary stabilization compared to a previously described closed fracture model with regular healing.

Surgical procedure of rabbit tibiae osteotomy as performed in this study generates a unique phenotype of bone defect according to the AO- classification for fractures (42A3). Hence, it provides consistent preconditions for further investigations.

Radiological, biomechanical and histological screening during the observation showed a clear course of impaired bone healing. Even after $84 \mathrm{~d}$ the osteotomized tibiae were far from the stage of remodeling the original bone structure. At best, defect bridging occurred periosteally but not inter-fragmentary as seen in the fracture model [24]. Primary periosteal bridging is an integral part of the approximately 5 week bone healing process in rabbit and has been described as species-specific [10]. On the other hand, a contributory factor may be the use of the intramedullary stabilisation technique. The development of delayed healing after tibial osteotomy in direct comparison to the closed fracture technique was previously documented in a rabbit model by Park et al. [11] using an external fixator for stabilization of the bone defect. These authors showed respective differences two weeks post bone defect, but these had disappeared at later observation time points. In a study with rats Kokubu et al. [12] developed a femoral model with $100 \%$ nonunion up to $56 \mathrm{~d}$ post surgery. However, as an effect of cauterization on each side of the fracture, the radiographical appearance of nonunions was atrophic.

Compared to our previously reported study in rats which underwent closed tibial fracture with the same intramedullary stabilization, clear differences in the biomechanical properties of the osteotomized tibiae could be observed in contrast to the fractured tibia. The osteotomized tibiae showed significant lower values for maximum torque and torsional stiffness after 42 and also 84 days as compared to the fractured tibiae. Even though sample preparation for biomechanical testing at an earlier time point $(28 \mathrm{~d})$ was not possible for all osteotomized bone samples, mainly due to the very unstable callus, the results were in accordance with those in 
a study from Shefelbine et al.[13] demonstrating also very low biomechanical data $21 \mathrm{~d}$ after rat tibial osteotomy.

Although our results can only be interpreted cautiously because of the small sample size investigated, the comparative examination of osteotomized and fractured bones in the present study gave evidence, that the course of delayed healing in osteomized animals is determined already before day 28 after surgery.

Histological investigations comparing the two different techniques even in earlier healing phases, at 28 d post bone defect, also revealed differences: The histomorphometric analyses showed that the amounts of accumulated desmal formed reparative bone tissue and of differentiated cartilaginous tissue were retarded in the region of the osteotomy defect compared to the fracture model.

Less deposition of trabecular bone could be found on the anterior tibial side for both groups. One compounding parameter may be the weakly developed soft tissue envelope on the anterior tibial side. Moreover, comparison of the regional accumulation of differentiated cartilage revealed that osteotomized animals accumulate lower amounts of differentiated cartilaginous cells on the anterior side where the surgical approach was located. We therefore conclude that the choice of surgical approach can influence the local formation of reparative cartilaginous tissue. Furthermore, in the callus of osteotomized rabbit tibiae we found numerous amounts of inflammatory cells. Additionally the number of vessels in callus was significantly higher than in the callus of fractured tibiae, where the amount decreased between 7 and $28 \mathrm{~d}$ post intervention. Comparable results i.e. an initial relatively constant number of vessels in the callus of tibiae with induced impaired healing in contrast to a decreased number in the regular healing group were shown in osteotomized sheep [14]. We would interpret these observations in the present study as a prolonged and pronounced inflammatory reaction occurring in all osteotomized rabbits. In fact, microbiological screening revealed persistent subclinical infection in a subset of the osteotomized animals. This can hardly be avoided given the keeping conditions and activities of the laboratory animals. For a rabbit model, Melcher and colleagues [15] showed even higher infection rates. Thus, tibial osteotomy has a higher chance of infection than closed fracture, something that also occurs in surgical treated closed tibial fractures in humans [1].

What could be a reasonable explanation for the delayed bone healing in all osteotomized animals in this study Radiologically, we could rule out poor end-to-end contact of osteotomy gap. Another frequently quoted cause could be a high degree of instability in the osteotomized bone, concerning the rotation movement [16]. But all animals were stabilized in the same manner as described for the healing fracture model and breed, weight and sex were analogous. Although this was not examined in any comparative measurements, differences in instability were not obvious in either group. However, variations within minimal inter-fragmentary movements cannot be ruled out. In fractured animals we observed the deposition of more defect-associated cartilage, pointing to a possible higher degree of instability capable of modulating the healing process [17]. The comparative analysis of tibia and fibula in osteotomized animals may support this hypothesis as more cartilaginous tissue formation was detected around the fractured and unstabilized fibula than around the stabilized tibial gap in histological bone slices from the same animal. However, the fibula was broken manually and the tibia by osteotomy. Both these parameters can influence the outcome.

Poor vascularization could be a plausible reason for non-union. The calluses appeared hypertrophical radiologically and well vascularised histological. Bone necroses at the fragment sides were not observed and severe injuries of the bone's nutrient vessels were not conspicuous during surgery. Furthermore, proliferating mesenchymal stem cells from bone marrow are though to play a key role in bone repair [18]. Primarily used for cooling purposes, open techniques require irrigation and it cannot be ruled out that progenitor cells were washed out. However, hematomas removal within the first hour was shown to be of no relevance for the healing progress [19]. The most plausible explanation for nonunion in this study may be an extended spatio-temporal inflammation and granulation phase, identifiable by dimensions, prolonged inflammatory cell composition and initial vascularization. Local infection as detected in a subset of animals may be a contributory factor but aseptic inflammation leading to nonunion [20] could be assumed to play a main role in our model.

As known from in vitro cell culture systems, environmental changes have the potential to influence the differentiation and functioning of cells. Regardless of the importance of inflammation for initializing the healing process, severe soft tissue trauma and the linked excessive release of inflammatory mediators can be discussed as factors to have a negative impact on bone healing [21]. Irrespective of the initial trigger, consideration should be given to whether persistent early inflammation could be a basic reason for nonunions.

\section{Conclusion}

The open tibial osteotomy technique combined with intramedullary stabilization in animals leads to pronounced impairment of bone healing. Prolonged local initial inflammation is suggested as a causative mechanism. We conclude that the reported osteotomy model is not suitable to provide insight into normal bone healing. Nevertheless, it could be used as a delayed healing model. 


\section{References}

[1]. Coles CP, Gross M: Closed tibial shaft fractures: Management and treatment complications. A review of the Prospective Literature. Can J Surg 2000, 43(4):256-262.

[2]. Auer JA, Goodship A, Arnoczky S, Pearce S, Price J, Claes L, von Rechenberg B, Hofmann-Amtenbrinck M, Schneider E, MüllerTerpitz R, Thiele F, Rippe KP, Grainger DW: Refining animal models in fracture research: seeking consensus in optimising both animal welfare and scientific validity for appropriate biomedical use. BMC Musculoskelet Disord 2007, 8:72.

[3]. Hietaniemi K, Peltonen J, Paavolainen P: An experimental model for non-union in rats. Injury 1995, 26:681-686.

[4]. Simon AM, Manigrasso MB, O'Connor JP: Cyclo-oxygenase 2 function is essential for bone fracture healing. J Bone Miner Res 2002, 17:963-976

[5]. Naimark A, Miller K, Segal D, Kossoff J: Nonunion. Skeletal Radiol 1981, 6:21-25.

[6]. Marsh DR, Li G: The biology of fracture healing: optimizing outcome. Br Med Bull 1999, 55:856-869.

[7]. O'Loughlin PF, Morr S, Bogunovic L, Kim DA, Park B, Lane JM: Selection and development of preclinical models in fracturehealing research. J Bone Joint Surg Am 2008, 90(Suppl 1):79-84.

[8]. Hiltunen A, et. al; A standardized experimental fracture in the mouse tibia. J orthop Res. 1993; 11 (2): $305-12$.

[9]. Schmidmaier G, Wildemann B, Melis B, Krummrey G, Einhorn TA, Haas NP, Raschke M: Development and characterization of a standard closed tibial fracture model in the rat. European Journal of trauma 2004, 30:35-42.

[10]. Wray JB, Lynch CJ: The vascular response to fracture of the tibia in the rat. J Bone Joint Surg Am 1959, 41-A:1143-1148

[11]. Park SH, O'Connor K, Sung R, McKellop H, Sarmiento A: Comparison of healing process in open osteotomy model and closed fracture model. J Orthop Trauma 1999, 13:114-120.

[12]. Kokubu T, Hak DJ, Hazelwood SJ, Hari Reddi A: Development of an atrophic non-union model and comparison to a closed healing fracture in rat femur. J Orthop Res 2003, 21:503-510.

[13]. Shefelbine SJ, Augat P, Claes L, Beck A: Intact fibula improves fracture healing in a rat tibia osteotomy model. J Orthop Res 2005, 23:489-493.

[14]. Lienau J, Schell H, Duda GN, Seebeck P, Muchow S, Bail HJ: Initial vascularization and tissue differentiation are influenced by fixation stability. J Orthop Res 2005, 3:639-645.

[15]. Melcher GA, Metzdorf A, Schlegel U, Ziegler WJ, Perren SM, Printzen G: Influence of reaming versus nonreaming in intramedullary nailing on local infection rate: experimental investigation in rabbits. J Trauma 1995, 39(6):1123-1128.

[16]. Mølster AO, Gjerdet NR: Effects of instability on fracture healing in the rat. Acta Orthop Scand 1984, 55:342-346.

[17]. Ashhurst DE: The influence of mechanical conditions on the healing of experimental fractures in the rabbit: a microscopical study. Philos Trans R Soc Lond B Biol Sci 1986, 313:271-302.

[18]. Bruder SP, Jaiswal N, Ricalton NS, Mosca JD, Kraus KH, Kadiyala S: Mesenchymal stem cells in osteobiology and applied bone regeneration. Clin Orthop Relat Res 1998, 355(Suppl):S247-256.

[19]. Grundnes O, Reikerås O: The importance of the hematoma for fracture healing in rats. Acta Orthop Scand 1993, 64:340-342.

[20]. Green SA, Moore TA, Spohn PJ: Nonunion of the tibial shaft. Orthopedics 1988, 11:1149-1157.

[21]. Bunn JR, Canning J, Burke G, Mushipe M, Marsh DR, Li G: Production of consistent crush lesions in murine quadriceps muscle-a biomechanical, histomorphological and immunohistochemical study. J Orthop Res 2004, 22:1336-1344.

Table-1. Biomechanical characterization of the osteotomized tibiae (torsional load and stiffness, as \% of contralateral intact tibia, mean \pm SD) compared to previously collected data for the fracture model.

\begin{tabular}{|l|l|l|l|}
\hline Treatment & $\mathrm{n}=$ & Torsional load & Stiffness \\
\hline Osteotomy 28d & 4 & $13.7 \pm 6.4$ & $12.1 \pm 7.7$ \\
\hline Fracture 28d & 7 & $53.0 \pm 21.0$ & $71.4 \pm 24.6$ \\
\hline Osteotomy 42d & 7 & $27.4 \pm 14.8$ & $17.0 \pm 13.2$ \\
\hline Fracture 42d & 8 & $117.3 \pm 32.6 *$ & $87.1 \pm 28.6 *$ \\
\hline Osteotomy 84d & 6 & $58.4 \pm 18.6$ & $22.2 \pm 11.9$ \\
\hline Fracture 84d & 8 & $176.2 \pm 61.8^{*}$ & $143.2 \pm 51.1 *$ \\
\hline
\end{tabular}

Table-2 Dimension of reactive callus $\left(\mathrm{mm}^{2}\right)$ along the tibiae of osteotomized rabbits compared to fractured animals 5 and 10 days post surgery (mean \pm SD) in X-rays.

\begin{tabular}{|l|l|l|l|}
\hline Dimension in & whole callus & callus anterior & callus posterior \\
\hline Osteotomy 7d & $14.7 \pm 5.6$ & $11.2 \pm 4.7$ & $3.2 \pm 0.7$ \\
\hline Fracture 7d & $11.6 \pm 1.7$ & $8.3 \pm 1.7$ & $3.1 \pm 1.2$ \\
\hline Osteotomy 28d & $24.8 \pm 9.3^{*}$ & $14.7 \pm 6.3^{*}$ & $10.8 \pm 2.8$ \\
\hline Fracture 28d & $14.9 \pm 3.1 *$ & $8.2 \pm 2.1$ & $6.7 \pm 1.6$ \\
\hline \multicolumn{4}{|r}{$* \mathrm{P}<0.05$ significant }
\end{tabular}

$* \mathrm{P}<0.05$ significant

Table-3- Amount of vessels (per $\mathrm{mm}^{2}$ ) in the callus (ROI) of osteotomized rabbits compared to fractured animals (mean $\pm \mathrm{SD}$ ) by histomorphometry.

\begin{tabular}{|l|l|l|l|}
\hline \multicolumn{1}{|c|}{ Amount in } & whole callus & callus anterior & callus posterior \\
\hline Osteotomy 7d & $49.6 \pm 12.8$ & $40.2 \pm 17.7$ & $34.6 \pm 19.6$ \\
\hline Fracture 7d & $41.1 \pm 13.2$ & $48.2 \pm 21.1$ & $37.6 \pm 4.3$ \\
\hline Osteotomy 28d & $38.5 \pm 12.8^{*}$ & $47.1 \pm 21.2^{*}$ & $14.6 \pm 6.8$ \\
\hline
\end{tabular}


Osteotomy Model Is Not Suitable To Provide Insight Into Normal Bone Healing

\begin{tabular}{|l|l|l|l|}
\hline Fracture 28d & $15.0 \pm 4.8 *$ & $18.2 \pm 4.1 *$ & $11.5 \pm 7.2$ \\
\hline \multicolumn{4}{|c|}{$* \mathrm{P}<0.05$ significant }
\end{tabular}

Table-4 Formation of newly formed bone in \% of reactive callus (ROI) in osteotomized rabbits compared to fractured animals 7 and 28 days post surgery (mean \pm SD).

\begin{tabular}{|l|l|l|l|}
\hline \multicolumn{1}{|c|}{ Amount in } & whole callus & callus anterior & callus posterior \\
\hline Osteotomy 7d & $7.2 \pm 9.6$ & $8.2 \pm 14.5$ & $8.7 \pm 9.8$ \\
\hline Fracture 7d & $8.9 \pm 7.2^{*}$ & $4.6 \pm 5.7^{*}$ & $16.4 \pm 11.3$ \\
\hline Osteotomy 28d & $17.2 \pm 8.4^{*}$ & $7.5 \pm 7.3^{*}$ & $26.2 \pm 9.1$ \\
\hline Fracture 28d & $34.8 \pm 8.0 *$ & $24.9 \pm 7.8^{*}$ & $48.8 \pm 16.2 *$ \\
\hline \multicolumn{4}{|r}{$* \mathrm{P}<0.05$ significant }
\end{tabular}

Table-5 Formation of cartilaginous tissue in \% of the callus (ROI) in osteotomized rabbits compared to fractured animals 7 and 28 days post surgery (mean \pm SD).

\begin{tabular}{|l|l|l|l|}
\hline Formation in & whole callus & callus anterior & callus posterior \\
\hline Osteotomy 7d & $0.4 \pm 0.13$ & $0.5 \pm 0.05$ & $0.3 \pm 0.01$ \\
\hline Fracture 7d & $3.37 \pm 1.4^{*}$ & $1.9 \pm 1.22^{*}$ & $1.87 \pm 0.07$ \\
\hline Osteotomy 28d & $10.2 \pm 6.4^{*}$ & $3.6 \pm 1.9^{*}$ & $15.7 \pm 3.7$ \\
\hline Fracture 28d & $28.1 \pm 6.4^{*}$ & $23.8 \pm 9.01 *$ & $16.6 \pm 7.8$ \\
\hline
\end{tabular}

$* \mathrm{P}<0.05$ significant
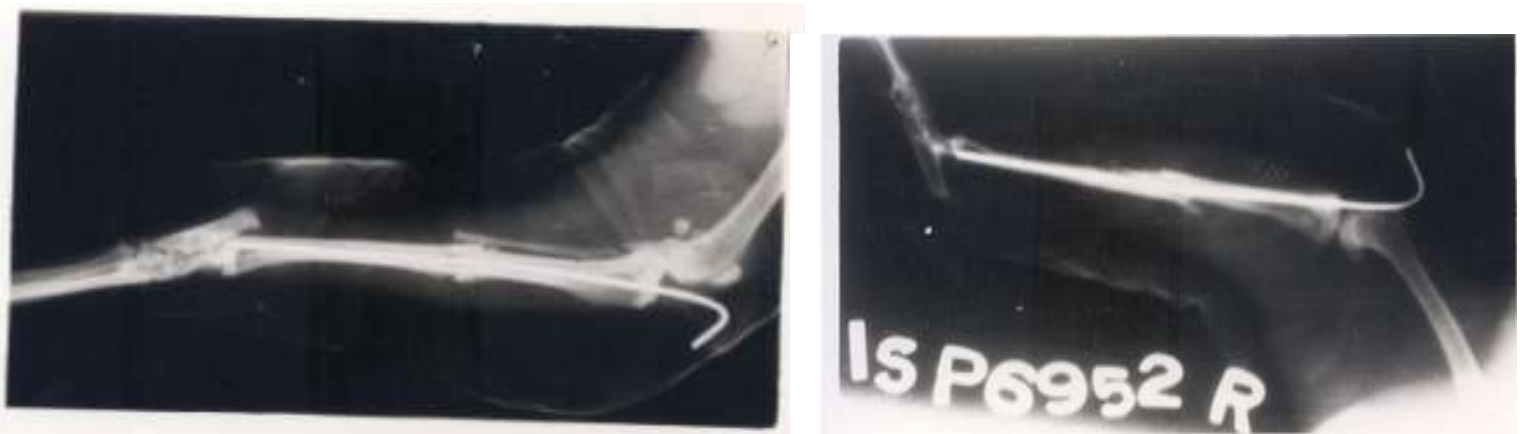

Figure1. Radiographs of a rabbit tibia after osteotomy (a) or fracture (b) over the complete observation period: radiographs of osteotomized animal revealed extremely impaired healing and clearly indicated nonunion.
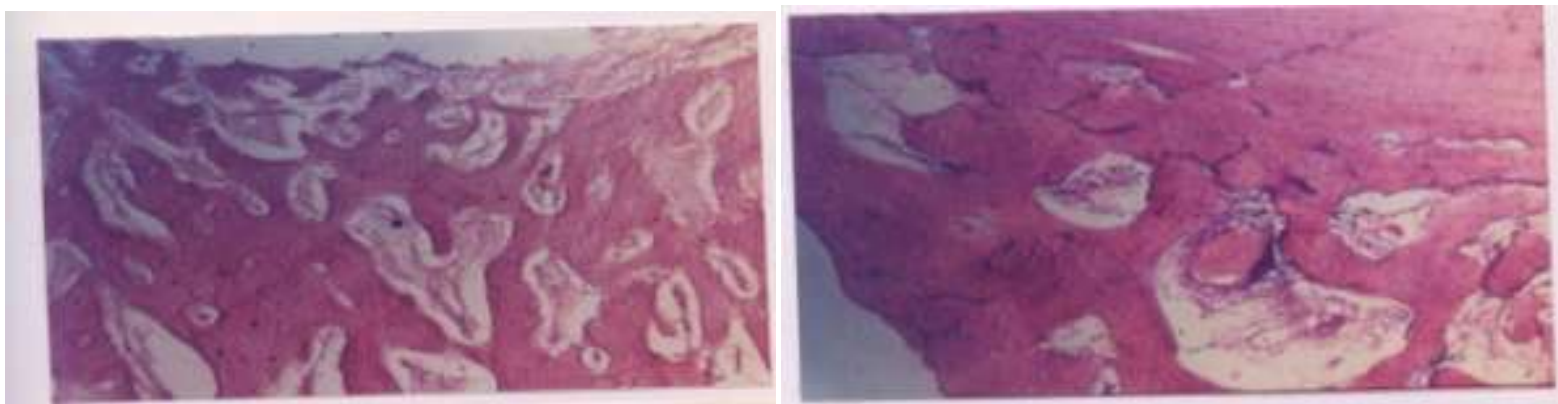

Figure 2. HE stained slices of comparable osteotomized (a) and fractured tibia areas (b) $28 \mathrm{~d}$ after surgery. 